\title{
The coding and inter-manual transfer of movement sequences
}

\author{
Charles H. Shea ${ }^{1 *}$, Attila J. Kovacs ${ }^{2}$ and Stefan Panzer ${ }^{3}$ \\ 1 Human Performance Laboratories, Department of Health and Kinesiology, Texas A\&M University, College Station, TX, USA \\ 2 Center for Complex Systems and Brain Sciences, Florida Atlantic University, Boca Raton, FL, USA \\ 3 Institute of Sport Sciences, Muenster University, Muenster, Germany
}

\section{Edited by:}

David L. Wright, Texas A\&M University, USA

\section{Reviewed by:}

Mathias Hegele, Justus Liebig

University Giessen, Germany

Raju Surampudi Bapi, University of

Hyderabad, India

\section{*Correspondence:}

Charles H. Shea, Department of Health and Kinesiology, Texas A\&M University,

College Station, TX 77843-4243, USA.

e-mail:cshea@tamu.edu

\begin{abstract}
The manuscript reviews recent experiments that use inter-manual transfer and inter-manual practice paradigms to determine the coordinate system (visual-spatial or motor) used in the coding of movement sequences during physical and observational practice. The results indicated that multi-element movement sequences are more effectively coded in visual-spatial coordinates even following extended practice, while very early in practice movement sequences with only a few movement elements and relatively short durations are coded in motor coordinates. Likewise, inter-manual practice of relatively simple movement sequences show benefits of right and left limb practice that involves the same motor coordinates while the opposite is true for more complex sequences. The results suggest that the coordinate system used to code the sequence information is linked to both the task characteristics and the control processes used to produce the sequence. These findings have the potential to greatly enhance our understanding of why in some conditions participants following practice with one limb or observation of one limb practice can effectively perform the task with the contralateral limb while in other (often similar) conditions cannot.
\end{abstract}

Keywords: movement sequences, effector transfer, coordinate system, sequence coding

\section{THE CODING AND INTER-MANUAL TRANSFER OF MOVEMENT SEQUENCES}

How movement sequences are represented and processed in the central nervous system has garnered a great deal of experimental and theoretical attention in the last 20 years. For example, Keele et al. (1995) and Verwey (1995) have proposed that movement sequences are processed in two independent coding/processing schemes which were labeled cognitive and motor. Cognitive processing was thought to be involved in the organization of the movement elements into subsequences (termed chunks by Verwey, 1994), linkages between subsequences (concatenation), and coding of the sequential spatial locations of the movement sequence. Motor processing was thought to be involved in selecting effectors and computing activation patterns to achieve the desired sequence of spatial locations. Both theoretical perspectives argued that cognitive and motor processing (or representations) were developed independently, at different rates during practice, and that different movements may rely differentially on these representations/processing types. For example, key press tasks are often used to understand the cognitive processing involved in sequence production because the motor demands are reduced because the onset of an effector has to be controlled to depress the key, but the specific amount, pattern, or coordination of agonist and antagonist forces does not have to be carefully regulated. In many other movements the precise regulation of forces across effectors have to be carefully managed in order to produce the correct movement pattern. Thus, the development of motor codes may progress faster for key press tasks than for tasks requiring the more precise control of forces. Numerous recent studies using a variety of tasks have found patterns of effector transfer that are consistent with these proposals (e.g., Schmidt, 1975; Willingham et al., 2000; Grafton et al., 2002; Park and Shea, 2002, 2003a,b, 2005; Whitacre and Shea, 2002; Verwey and Clegg, 2005; van Mier and Petersen, 2006).

Using a different approach Jordan (1995) proposed the notion of dynamic optimization of motor codes/processing. He used a relearning paradigm with skilled typists on an altered keyboard. Performance decrements observed during relearning using the altered keyboard suggested that through extensive typing practice with a traditional QWERTY keyboard the typists had developed an effector dependent representation of the spatial locations of specific keys. That is, an optimized response had been developed where specific spatial locations were linked to specific effector movements. This optimization was thought to result when neurological and anatomical properties of a specific effector system are exploited through practice in order to enhance response production (Park and Shea, 2005) or when a coarticulation mechanism tunes activation patterns of specific effectors to the biomechanical properties of that effector (Verwey and Clegg, 2005). According to the notion of dynamical optimization, with increased amount of practice a response becomes increasingly effector specific due to the specific effector information that is being coded along with and perhaps linked to sequence information. In Keele's and Verwey's terms the cognitive and motor levels of response production became linked in such a way that they no longer maintain their independence or the motor commands have been so refined that additional cognitive processing is not required during the production of the movement sequence. The result is that the optimized response sequence is more specific to the precise conditions experienced during the optimization period of practice, but becomes increasingly more inflexible under transfer conditions as practice continues. Indeed, recent sequence learning experiments looking at effector, force, and 
spatial transfer after one and multiple days of practice have found that the transfer surface becomes increasing limited over extended practice (e.g., Jordan, 1995; Park and Shea, 2003b, 2005; BischoffGrethe et al., 2004; Wilde and Shea, 2006; Wilde et al., 2008).

Hikosaka et al. $(1999,2002)$ proposed that the processing of a movement sequence is distributed in the brain in independent spatial (e.g., spatial locations of end effectors and/or sequential target positions) and motor (e.g., sequence of activation patterns of the agonist/ antagonist muscles and/or achieved joint angles) coordinate systems with different neural substrates subserving each class of processing. According to this perspective the learning of movement sequences involves both a fast developing, effector independent component represented in visual-spatial coordinates, and a slower developing effector dependent component that is represented in motor coordinates. In our opinion this perspective has the potential to provide with some modifications a unifying way to understand the various factors that influence coding and transfer of movement sequences.

In terms of the neural substrate, the Hikosaka et al. (1999) model proposed that intracortical bidirectional (loop circuits) connections develop over practice between the association cortices, motor cortex, basal ganglia, and cerebellum. Visual-spatial processing is supported by circuits formed between the prefrontal and parietal cortices, anterior basal ganglia (head of the caudate), and posterior lobe of the cerebellum while motor processing is supported by the motor cortex, midposterior basal ganglia (putamen), anterior lobe of the cerebellum, and dentate nucleus circuits. The spatial and motor mechanisms are capable of operating independently, and thus, through practice an individual acquires a given sequence in visual-spatial and motor coordinates. For successful completion of the sequential task the two mechanisms must interact. This interaction is facilitated in two ways: a translation mechanism relying predominantly on the premotor area, and a coordination or switching mechanism relying predominantly on the pre-supplementary motor area (pre-SMA). The role of the translation mechanism during initial stages of practice is to transform the information from visual into motor coordinates while the role of the coordinative mechanism is to suppress the output of the motor sequence mechanism if this output conflicts with that of the spatial sequence mechanism. The imaging work suggests the association cortex, anterior basal ganglia circuits, and parietal-prefrontal cortical loops are more active early in learning. During this early stage of learning explicit knowledge related to the visual-spatial characteristics of the sequence seem to be available to consciousness and attention requirements are relatively high. On the other hand, the circuits within the motor system appear to develop more slowly and at a more implicit level. Hikosaka proposed that eventually practice results in a shift from loops specific to visual-spatial coordinate processing to loops associated with motor coordinate processing.

According to this perspective the two sequential processes are developed in parallel, each coded in a different coordinate system. Initially, a sequence is coded in visual-spatial coordinates that rely on attention, explicit knowledge and working memory. The visualspatial representation is thought to be transferable to unpracticed effectors resulting in relatively good performance of a novel task variation that has the same visual/spatial characteristics. In parallel, another code represented in motor coordinates (e.g., sequential pattern of muscle activation and/or joint angles) also develops.
Motor representations are more effector specific (Hikosaka et al., 2002) given that anatomical and neurological properties of the specific effector used during practice are being exploited to improve performance (Jordan, 1995; Park and Shea, 2005), and thus transfer to other effectors based on this code would be limited.

The model proposed by Hikosaka et al. $(1999,2002)$ was developed based largely on findings from multi-element key pressing tasks (e.g., $2 \times 5,2 \times 10$ ). The $2 \times 5$ and $2 \times 10$ tasks were originally devised to test sequence learning in monkeys (Hikosaka et al., 1995) and later humans (e.g., Hikosaka et al., 1996; Sakai et al., 1998; Bapi et al., 2000). The $2 \times 10$ task in the Bapi et al. (2000) experiment, for example, required participants to complete trials (termed hypersets) composed of 10 sets where each set involves sequentially depressing two keys on the key pad of the computer keyboard. The trial starts with the illumination of two squares on a $3 \times 3$ grid and the participant sequentially "hits" the corresponding keys on the $3 \times 3$ keyboard (see Figure 1). The first finger was to be used to depress the keys on the left column, middle finger the keys in the center column, and the ring finger for the keys on the right column. If the participant depressed the keys in the wrong order (where the correct order was learned by trial and error), the correct keys were not depressed, and/ or the response was not entered in $1.2 \mathrm{~s}$ the same set was repeated. If the set was responded to correctly and within the "time out window," the next set was presented. The trial was completed when the participant performs the 10 sets without an error and a training block was completed when four trials were completed successfully.

Test blocks were provided after 1 training block (early stage), after 2 training blocks (intermediate stage), and after 11 training blocks (late stage). Test blocks were conducted in a manner similar to that of the training block (normal) except that the set was not redone when an error was made. Testing involved a normal condition in which the hand was positioned on the keyboard as it was during training (hand extending from the bottom of the display with the fingers pointing up to the keyboard) and two transfer blocks where the hand was rotated counter clockwise $90^{\circ}$ (hand extending from the left of the keyboard with the fingers pointing to the left (Figure 1). One test was termed a spatial ${ }^{1}$ test because the illuminated squares appeared in the same spatial position as during training but because of the new hand position different fingers had to be used to execute the response. Another test was termed motor because the position of the illuminated squares were altered ( shifted $90^{\circ}$ ) so that the same pattern of finger movements were required to produce the correct response to each set. At the early stage of practice performance was similar on the visual and motor tests but as practice increased performance on the motor transfer tests increasingly improved over that on the visual-spatial tests (intermediate and late stages). Based on these types of tasks the model predicts that the reliance on the visualspatial representation will gradually decrease over practice. Later in practice the production of the sequential movements rely more

${ }^{1}$ In an attempt to use consistent labeling, we will refer to the tests as retention, (same conditions as during practice - motor and spatial coordinates reinstated), motor (transfer with motor coordinates reinstated), and spatial (transfer with spatial coordinates reinstated). In the literature these tests have been referred to with various labels. For example, Bapi et al. (2000) referred to the retention test as the "normal" test. Similarly, Kovacs et al. (2009b) and Boutin et al. (2010), have referred to the motor and spatial tests as "mirror" and "non-mirror," respectively and Lange et al. $(2004,2006)$ referred to the retention test as the "learned" condition and the motor and spatial test as "mirror" and "normal" transfer tests, respectively. 


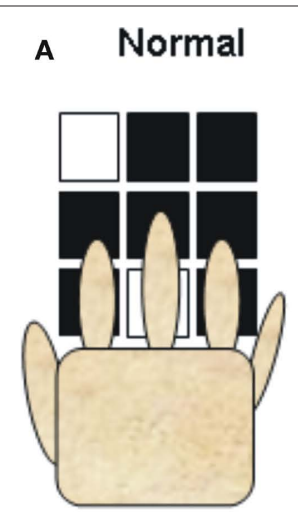

B Normal

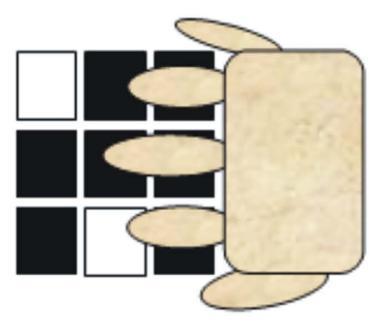

Spatial
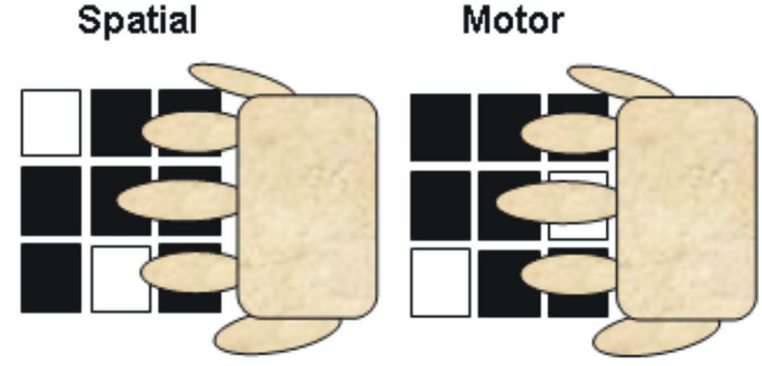

Spatial

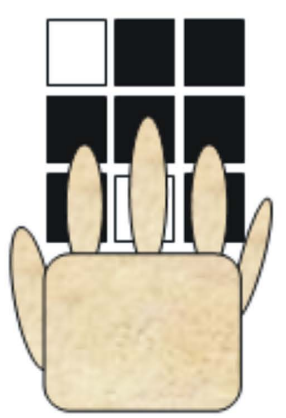

Motor

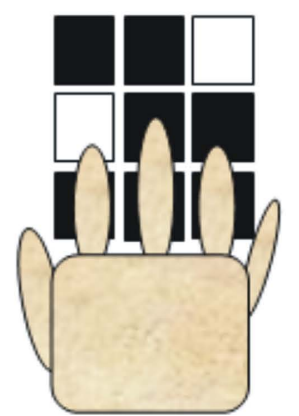

FIGURE 1 | Illustration of the hand position and finger movement in two versions $(A, B)$ of the normal (acquisition and retention), visual, and motor conditions. In the visual condition the display is to the same as in the normal condition, but because the hand position is changed different fingers and movements are required to move to the correct keys. In the motor condition the display is changed from the normal condition, but because the hand position has changed the same fingers and movements are required. Note that the illustration only depicts a single set. In the $2 \times 10$ tasks, a trial was composed of 10 sets. (redrawn from Bapi et al., 2000). heavily on the motor representation which allows a more rapid and precise execution of the sequence. It should be noted, however, that the processing demands and therefore the representations for key press sequences may differ in important but subtle ways from that of continuous movement sequences. Key press sequences involve a sequential pattern of muscle activation, but the precise regulation of forces and the management of movement dynamics is not required to the same extent as required in many movement sequences where a specific pattern of flexion and extension movements is required. This difference may play a role in determining the effectiveness of codes represented in visual-spatial and motor coordinates across practice and introduce additional factors that must be considered in determining the most effective coding scheme.

Recently, inter-manual transfer (e.g., Kovacs et al., 2009a,b, 2010), inter-manual practice (e.g., Panzer et al., 2009a,b, 2011), and observational learning (e.g., Boutin et al., 2010; Gruetzmacher et al., 2011) research looking at the coding of simple and complex movement sequences have offered some interesting modification to the Hikosaka perspective. Note that these sequences require the precise control of agonist and antagonist muscle groups and the management of the movement dynamics in order to precisely regulate forces as well as store and utilize elastic forces and momentum during movement reversals that is not required when discrete key presses are utilized. In fact, as noted earlier key press tasks have often been used to study the cognitive processes involved in sequence production because these processes are more easily isolated when the motor demands of the task are reduced. Importantly, the results of these recent experiments suggest that the Hikosaka perspective should be modified in two ways. First, these experiments suggest that codes developed in motor coordinates can be transferred to homologous muscles on the contralateral limb (mirror movements). Hikosaka et al. (1999) proposed that codes developed in motor coordinates were effector specific with practice resulting in codes which optimize the joint angles, kinematics, and dynamics of the practiced limb. Second, Hikosaka et al. (1999) proposed a transition from visual-spatial to motor codes over practice. These recent experiments suggest that the characteristics of the movements being studied and the associated control processes used to produce the response play a role in determining the time course of this transition such that relatively simple movement sequences that can be preplanned experience this transition very early in practice while longer duration multi-element movement sequences may be best coded in visual-spatial coordinates even after extended practice. These results offer the potential to explain why participants in some effector transfer experiment exhibit the ability to effectively transfer a task learned with one limb to the contralateral limb while in other tasks/conditions the ability to transfer to the unpracticed limb is quite limited. 
It should also be noted that the Hikosaka model has similarities to theoretical perspectives that propose intrinsic and extrinsic coordinate or coding systems (e.g., Krakauer et al., 1999; CriscimagnaHemminger et al., 2003). The intrinsic coordinates are thought to be represented in terms of an internal model of joint representations (Criscimagna-Hemminger et al., 2003), musculoskeletal forces and dynamics (Krakauer et al., 1999), and/or orientation of body segments relative to each other (Lange et al., 2004). This type of coordinate system is thought to be effector dependent to the extent that biomechanical, neurological, and dynamic properties of the effectors used on the transfer test are dissimilar to those used during practice. That is, an intrinsic representation was proposed to "generalize with mirror symmetry reflecting the joint's symmetry” (Criscimagna-Hemminger et al., 2003). Similarly, Lange et al. (2004) proposes that "Intermanual transfer of movements previously learned with one hand is accomplished by the recall of unmodified extrinsic coordinates if the task is performed in the original orientation. Intrinsic coordinates are retrieved in the case of mirror-reversed orientation." The extrinsic coordinates were thought to reflect Cartesian coordinates of the task space with respect to the body and visual display. This coordinate system was thought to be effector independent when the extrinsic coordinates are reinstated during an effector transfer test even though intrinsic characteristics of the required transfer movement may have been altered. Thus, the descriptions of intrinsic and extrinsic coordinates are similar to Hikosaka's description of motor and visual-spatial coordinates at least with respect to effector transfer.

The following sections will review recent experiments aimed at determining the influence of sequence complexity and control processes on the development of movement codes based in visual-spatial and motor coordinates. In addition, recent experiments aimed at determining the coordinate system used to code movement sequences during physical and observational practice are reviewed. Finally we summarize our perspective of these findings relative to the Hikosaka perspective and offer some additional factors that should be considered to have an influence on effector transfer.

\section{INTER-MANUAL TRANSFER: SEOUENCE COMPLEXITY}

Kovacs et al. (2009b) conducted three experiments in which the amount of practice was manipulated across experiments using a complex 14-element dynamic arm movement sequence
(Figure 2A). The task required the participants to move a lever as quickly and smoothly as possible to the illuminated target position by flexing or extending their arm at the elbow. Upon "hitting" the target (by moving the pointer attached to the lever into the target area) the illumination was turned off and the next target in the sequence was immediately illuminated. Ten repetitions of the 14-element sequences were provided in each block and each day of practice involved 16 blocks of practice. Over practice, participants appeared to impose a structure on the movement sequence resulting in increasingly rapid production of the sequence. In each experiment participants practiced the movement sequence with either the left or right limb for 1, 4, or 12 days (Experiments 1-3). Following the various amounts of practice, a delayed retention test (Figure 2B) and two effector transfer tests were administered. One transfer test (motor) involved the unpracticed limb with a mirror presentation of the target positions, which required the same pattern of homologous muscle activation and the achievement of the same relative joint angles as during practice with the contralateral limb, but the spatial locations were altered (Figure 2C). On the other effector transfer test (spatial) the visual-spatial coordinates were reinstated such that participants moved to the same spatial locations as during acquisition but the unpracticed limb was used (Figure 2D). However, because the contralateral limb was used, a new unpracticed pattern of muscle activation and joint angles were required to achieve the target locations in the sequence. Thus, during the motor transfer test, the motor coordinates were reinstated and the visual-spatial coordinates were altered, while during the spatial transfer test the visualspatial coordinates were reinstated and the motor coordinates were altered. The Hikosaka perspective would predict an increased reliance over practice on codes formulated in motor coordinates. Based on this notion, we predicted increasingly better transfer performance when the motor coordinates are reinstated on the transfer test relative to transfer when the spatial coordinates are reinstated as practice increased.

Results, however, indicated that regardless of the amount of practice participants performed substantially better on the spatial transfer test than on the motor transfer test. While additional practice may eventually result in better motor transfer than spatial transfer these results suggest, at a minimum that the amount of practice required to develop motor codes, which are more effective than the visual spatial codes, may dramatically increase when the
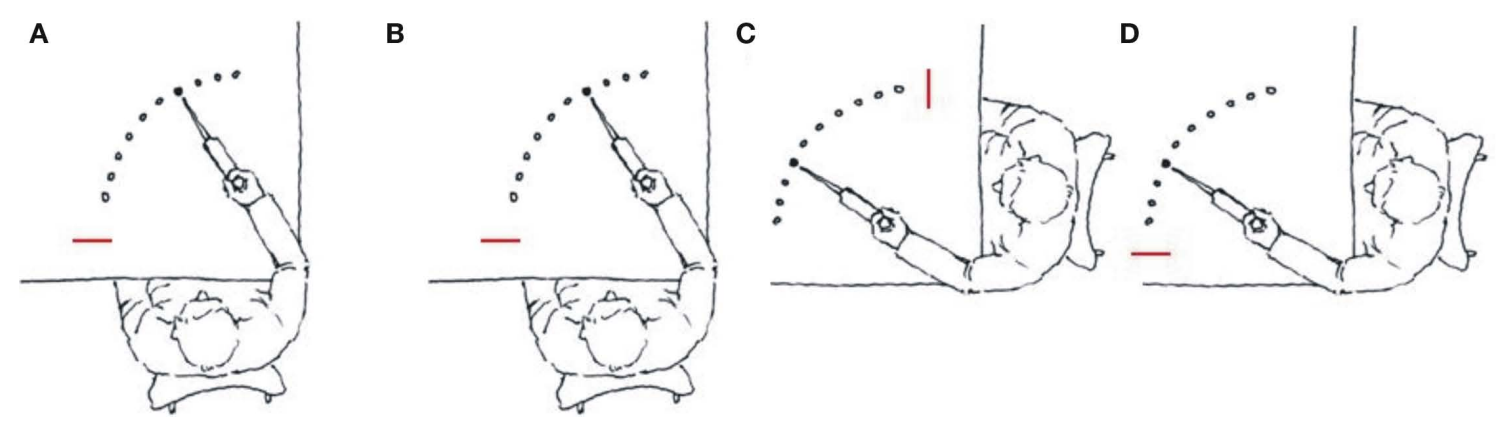

FIGURE 2 | Illustration of the arm used during acquisition (A) and on the retention (B), motor (C) and spatial (D) tests. Note that the targets were arbitrarily labeled 1-10 from the start position (red line). (redrawn from Kovacs et al., 2009b). 
complexity of the movement sequence increases. Indeed, this may even suggest that for some complex tasks that spatial coordinates may provide the optimal metric from which to develop movement codes independent of the amount of practice. Interestingly, the task used in the experiments by Kovacs et al. (2009b) has been shown to be structured by the participant into a series of concatenated subsequences (see Park and Shea, 2005). The processes of imposing a structure on a movement sequence with many elements is thought to reduce the processing and cognitive intervention in the control processes, but not eliminate dependence on cognitive processing. When a sequence is composed of fewer elements (e.g., Klapp, 1995), it may be possible to eliminate cognitive intervention during the production of the movement, but may not be possible for sequences with many elements even with extended practice.

Shea and colleagues (Kovacs et al., 2009a; Panzer et al., 2009a) hypothesized that relatively simple motor tasks may be coded, even following relatively little practice, in motor coordinates. To test this notion Kovacs et al. (2009a) asked participants to practice either a simple or a slightly more complex movement sequence for one practice session (99 trials). The simpler movement sequence ( $\mathrm{S} 1$ ) involved three movement reversals with a movement duration of $1300 \mathrm{~ms}$ and the more complex movement sequence (S2) involved five movement reversals with a movement duration of $2000 \mathrm{~ms}$ (see Figure 3). Interestingly, the first $1300 \mathrm{~ms}$ of the longer sequences was the same as the shorter duration sequence. While it was not possible to precisely manipulate movement difficulty or complexity, Kovacs et al. (2009a) hypothesized that the increased movement duration and number of reversals (functional difficulty) of S2 may result in participants more effectively coding the sequence using spatial coordinates following only 99 trials of practice while S1 may be coded at this stage of practice in motor coordinates.

The results confirmed these predictions. Participants that practiced S1 performed the motor transfer test with the contralateral limb as effectively as they performed the retention test, which was conducted under the same conditions and with the same limb as during practice. Alternatively, participants that practiced S2 performed the spatial transfer test, where the spatial coordinates were reinstated, as well as the retention test. These findings provide strong support for the notion that the coordinate system used to code movement sequences and the manner in which participants respond on effector transfer tests is influenced by the characteristics of the movement. Consistent with this notion, the harmonicity values for S1 were consistent with that typically found for pre-planned movements $(H=0.90)$. On the other hand, harmonicity values for S2 were consistent with participants using on-line control to make subtle corrections during the progress of the movement particularly in the later segment of the movement. Note that harmonicity (Guiard, 1993) ranges from 0 to 1 with values lower than 1 indicating more than 1 peak in the acceleration trace for each half cycle of movement with the value falling below 0.5 if the additional peak crosses from positive to negative (or vice versa). A value of $H=1$ indicates harmonic motion. In essence, the lower the value of $H$ the more hesitations and/or adjustments in the movement occur. This finding caused Kovacs et al. (2010) to hypothesize that the coordinate system used to code sequence information may be, at least to some extent, dependent on the




control processes used rather than the sequence characteristics per se. An experiment testing this hypothesis will be discussed in a following section.

It is also worth noting that in a strict sense these data are not contrary to the predictions of Hikosaka et al. (1999) model. It is possible that participants in the Kovacs et al. (2009a) and Panzer et al. (2009a) experiments, where relatively simple movement sequences were tested, had already transitioned from an initial coding scheme based on visual-spatial coordinates to codes based in motor coordinates even though only 99 trials of practice was provided before the tests were conducted. It is logical that this transition should occur early in practice for a relatively simple movement sequence. Indeed, it appears that Hikosaka et al. (1999) and Bapi et al. (2000) were aware that in simple tasks there may be a very quick transition from visual-spatial codes to motor codes. To extend the visual-spatial stage Bapi et al. $(2000,2006)$ introduced complex coordinate transformations ${ }^{2}$. Similarly, it should be recognized that participants in the Kovacs et al. (2009b) experiment where a 14-element movement sequence was practiced for 1,4 , or 12 days may have required additional practice before movement codes based on motor coordinates could develop to a point that these codes could produce the movement more effectively on the motor transfer test than that exhibited on the visual-spatial transfer test.

\section{INTER-MANUAL TRANSFER: CODING AND CONTROL}

As noted previously, Kovacs et al. (2010) hypothesized that the production of shorter duration, less complex movements, which appear to be controlled by pre-planned processes, may develop reliance on motor coordinates earlier in practice than longer duration movement sequences, which appear to be structured into a series of subsequences and which are thought to require on-line control for the production of the successive segments of the movement sequence (e.g., Park and Shea, 2005; Wilde and Shea, 2006). To test this notion, Kovacs et al. (2010) utilized a goal sequential movement with five reversals and 2000-ms duration. In one condition participants were provided a template indicating the goal pattern and a cursor indicating the current position of their limb movement prior to and during the movement. These conditions were designed to increase the likelihood that participants would engage in online control. A second condition was designed so that participants could view the goal movement template prior to responding but the template was removed as soon as they began to move and no cursor was provided. These conditions were designed to encourage participants to preplan the movement. In both conditions the goal pattern overlaid with the participant produced waveform were displayed as feedback following the completion of the movement. Note that participants in both conditions were asked to produce the same movement pattern during acquisition, were provided the same number of practice trials, and retention and transfer tests for the two groups were conducted in the same manner. Consistent with their initial predictions the findings indicated that participants in the online condition transferred better when the spatial coordinates were reinstated on the transfer test than when the motor coordinates were reinstated. Participants in the preplanned condition produced enhanced transfer when the

${ }^{2}$ We wish to thank an anonymous reviewer for providing this information. motor coordinates were reinstated. These results provide strong evidence that the control system used during response production can play a critical role in determining the coordinate system which results in smaller errors on the effector transfer tests.

Consistent with this notion is that pre-planning and on-line control of movement sequences have been shown to utilize different information and rely on different neural pathways (e.g., Hikosaka et al., 2002; Glover, 2004). In other words, shorter duration movements with few elements predominantly rely on pre-planning while longer duration movements with more elements have an initial pre-planned component after which movement control is gradually taken over by the on-line control mechanism. Indeed, Smiley-Oyen and Worringham (2001) have demonstrated that movement trajectory, average velocity, and number of targets influence planning. These findings suggest that sequential tasks with dissimilar spatiotemporal characteristics might also differentially rely on distinct control mechanisms and benefit from distinct coordinate systems in the coding of movement sequences.

\section{DESIGNING INTER-MANUAL PRACTICE TO ENHANCE RETENTION}

Using a different approach and paradigm Panzer et al. (2009a) and Panzer et al. (2011) proposed that inter-manual practice, where the right limb is used in the first practice session and the left limb is used in the second practice session or vice versa, would benefit from organizing the two practice sessions so that the coordinate system used to code the movement sequence at that stage of practice was reinstated during both practice sessions. That is, for example, if the code that produces the more effective response utilized motor coordinates then organizing the two practice sessions so that the same pattern of muscle activation and pattern of joint angles were required even though different limbs were used should result in enhanced learning. Similarly if the code is more effective when developed in visual-spatial coordinates then moving to the same visual-spatial locations in the left and right limb practice sessions should result in enhanced learning. In the Panzer et al. (2009a, Experiment 2) experiment the participant's task was to reproduce a waveform projected on the screen. The goal sequence involved three reversals with a $1300-\mathrm{ms}$ duration. Figure 4 provides an illustration of the paradigm for the relatively simple movement sequence used by Panzer et al. (2009a). Note that the motor coordinates are reinstated in Acquisition 2 for the $\mathrm{A}$ and $\mathrm{C}$ conditions while the spatial coordinates are reinstated in Acquisition 2 in the B and D conditions. For example, if the movement is best coded in a spatial coordinate system then learning will be enhanced when that coordinate system is reinstated in the second practice sessions. Further, they proposed that the retention of this relatively simple movement sequence will be enhanced when motor coordinates are maintained across sessions while the retention of more complex movement sequences involving additional reversals and/or increased movement time will be enhanced when both practice sessions involve the same spatial coordinate system.

Consistent with their predictions, Panzer et al. (2009a) found a relatively short duration (1300 ms) movement sequence with three reversals to result in enhanced retention when the two practice sessions involved the same motor coordinates. These results are consistent with a number of findings from the effector transfer 


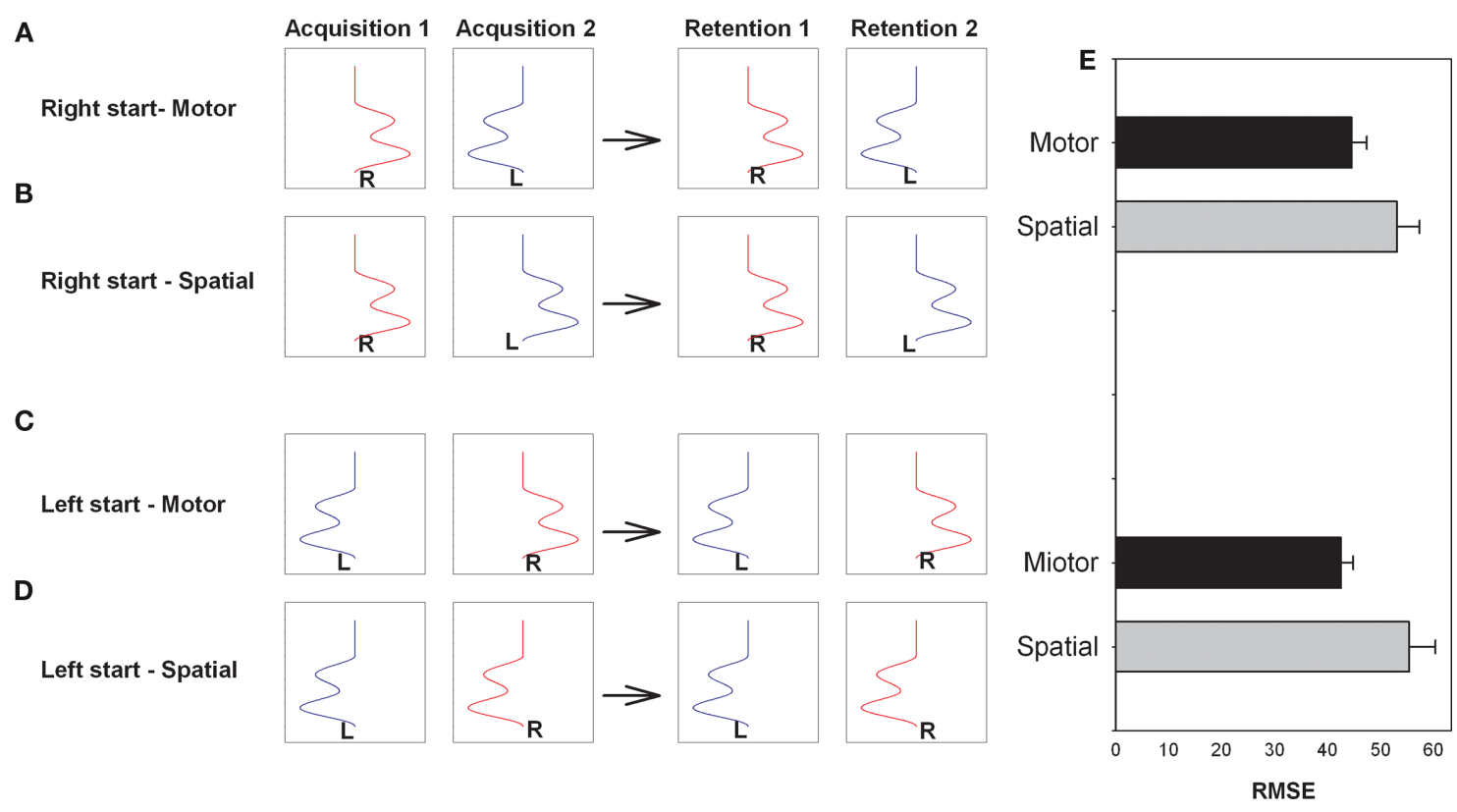

FIGURE 4 | Illustration of the arm and task used on acquisition sessions (1 and 2) and retention tests (1 and 2 ) for the right start group with the same motor coordinates on the two acquisition session (A) and right start group with the same spatial coordinates on the two acquisition sessions (B). Left start group with the same motor coordinates (C) and same spatial coordinates (D) are also illustrated. Retention performance is for each condition is provided to the right (E). Note that this design does not require effector transfer test to determine the coordinate system used to code the movement sequence (Redrawn from Panzer et al, 2009a).

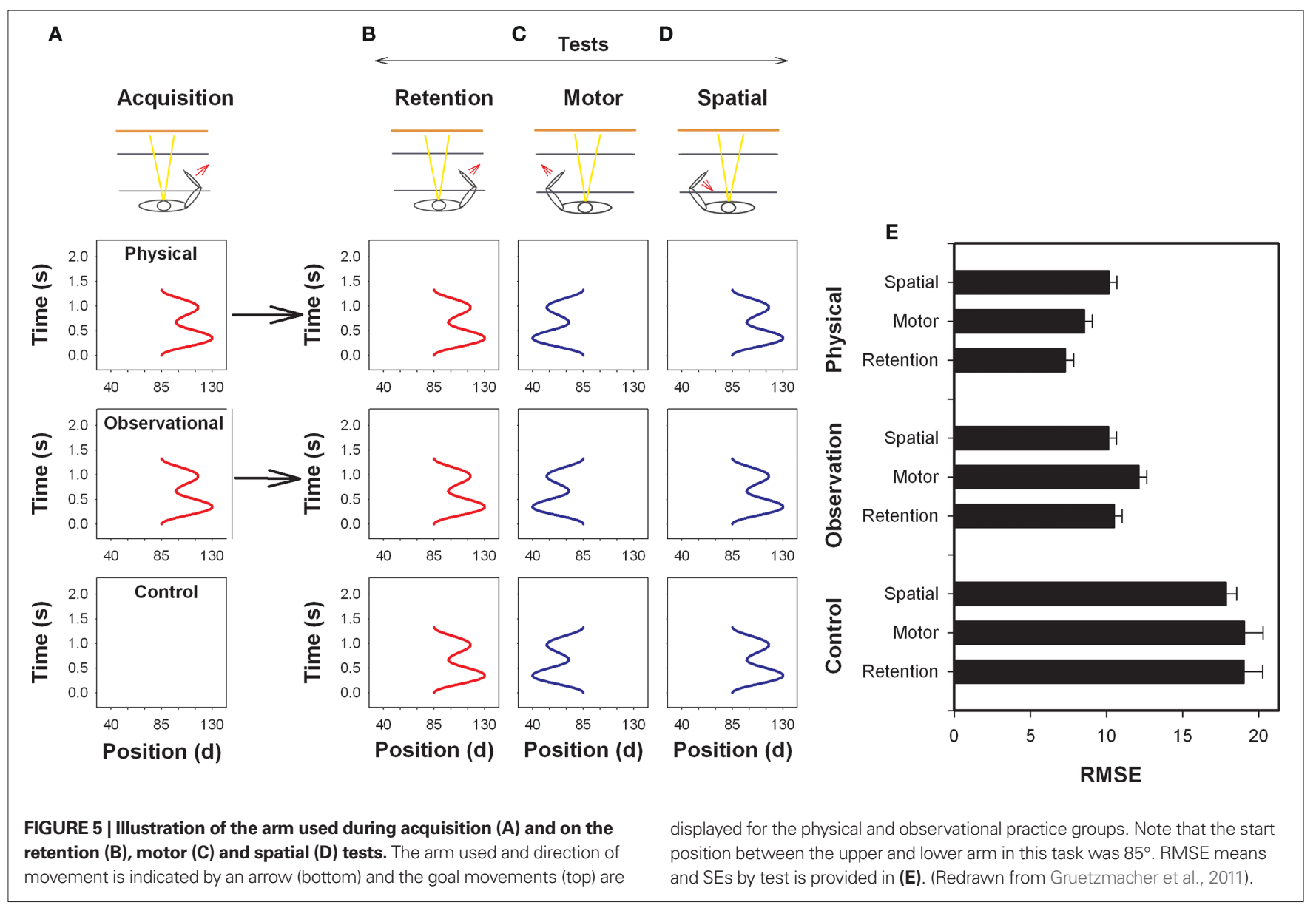


experiments discussed earlier and confirm that task characteristics play a role in determining the coordinate system used to code responses which results in the smaller errors on the effector transfer tests. Alternatively, when the task involved a more complex 16-element movement sequence, Panzer et al. (2009b) found inter-manual practice with the same spatial coordinates resulted in significantly better learning than inter-manual practice with the same motor coordinates (motor condition). It is particularly important that these findings are congruent with findings from experiments using interlimb transfer because in the inter-manual transfer experiments the experimenter has to rely on performance on transfer tests using the unpracticed limb which could mask any effector specific coding while in the inter-manual practice experiments only retention tests are required.

\section{INTER-MANUAL TRANSFER FOLLOWING OBSERVATIONAL PRACTICE}

A number of observational practice experiments have demonstrated that observation of a model performing a motor skill can facilitate the learning of a wide variety of motor tasks and many of these authors have argued that the representation and processing mechanisms developed during observation and later used when given the opportunity to physically practice are similar to those developed and used during physical practice (e.g., Blandin and Proteau, 2000; Bird and Heyes, 2005; Mattar and Gribble, 2005; also see Rizzolatti et al., 2001, for review). For example, Osman et al. (2005) using an eight element key press sequence found that participants could effectively produce the sequence with the limb they observed during observational practice but not with the contralateral limb (unobserved) suggesting that the movement sequence following observation was coded in motor coordinates. In addition, neuroimaging experiments report that a set of common neural structures are activated during both action production and action observation (e.g., Decety et al., 1997; Grèzes and Decety, 2001). Fadiga et al. (1995; also see Buccino et al., 2001) reported that "the pattern of muscle contraction evoked by transcranial magnet stimulation (TMS) during action observation is very similar to the pattern of muscle contraction present during the execution of the same action" (pp. 2609). These findings suggest a functional equivalence between action production and action observation. Indeed, a number of experiments have found that variables influencing physical practice also influence observational practice in a similar way (e.g., Wright et al., 1997; Shea et al., 2001; Badets and Blandin, 2004).

In a recent experiment, Boutin et al. (2010) looked at the coding of the complex 16-element movement sequence similar to that used by Kovacs et al. (2009b) following physical practice and action observation. An observer watched a video of a model that had not been exposed to the task before (often termed a learning model) and was privy to all instructions, observation of the movement and extrinsic feedback provided the model during physical practice. Consistent with their predictions, which were based on the earlier work with only physical practice conditions by Kovacs et al. (2009b), the physical practice participants performed the spatial transfer test as effectively as the retention test. Performance on the motor transfer test was significantly poorer. Interestingly, the observers although not permitted prior physical practice also performed the spatial transfer test as effectively as the retention test. For the observers, performance on the motor test was also significantly poorer than that on the retention and spatial tests.

In a similar experiment, Gruetzmacher et al. (2011) investigated physical and observational practice with the simple waveform reproduction task used earlier by Kovacs et al. (2009a). In the Kovacs et al. (2009a) experiment, they found participants after only one practice session to perform better on the motor transfer test. In the observational practice experiment Gruetzmacher et al. (2011) wanted to determine if observers would also initially code this response in motor coordinates (see Figure 5). This prediction was based on the finding that a common set of neural structures are activated during physical and observational practice (Decety et al., 1997; Grèzes and Decety, 2001) and the finding that factors that influence learning through physical practice appear to influence learning through observational practice (e.g., Wright et al., 1997; Shea et al., 2001; Badets and Blandin, 2004). If this were the case it would provide evidence that motor information can be extracted through observation. Consistent with previous findings (Kovacs et al., 2009a), they found that physical practice participants performed the motor transfer test better than the spatial but contrary to their initial predictions observers performed the spatial test better than the motor. Indeed, there was no difference between the observers and the physical practice participants' performance on the spatial test. The findings that coding appears similar for physical and observational learning when visual-spatial coordinates are reinstated on the transfer test but not when motor coordinates are reinstated may help explain why observation for some tasks results in retention and transfer performance that is as effective as that exhibited by physical practice participants while in other experiments observers perform more poorly than participants provided physical practice.

\section{GENERAL CONCLUSION}

Hikosaka et al. (1999) proposed that two sequential processes are developed in parallel, each coded in a different coordinate system. Initially, a sequence is coded in visual-spatial coordinates that rely on attention, explicit knowledge and working memory. In parallel, another code represented in motor coordinates that relies on implicit knowledge and does not require attention or working memory also develops. Early in practice participants rely on codes developed in visual-spatial coordinates but as practice progresses there is a shift to codes developed in motor coordinates. Recent experiments, however, suggest that both codes are developed through practice with one code typically resulting in better intermanual transfer depending on the amount of practice, the control processes used during practice, and the characteristics (complexity and/or difficulty) of the movement sequence. For example, in the extended practice experiments of Kovacs et al. (2009b) using a 14-element movement sequence, they found that regardless of the amount of practice $(1,4,12$ days of practice) that participants performed significantly better on the spatial effector transfer test (termed non-mirror test), where the visual-spatial coordinates were reinstated but the motor coordinates were changed, than on the motor effector transfer test, where the motor coordinates were reinstated and the visual-spatial coordinates were changed. 
They recognized that performance also improved on the motor transfer test with additional practice although the improvement was relatively small compared to that on the visual-spatial transfer test. Thus, it appeared that both codes continued to be refined over practice but the code based on motor coordinates was developing at a slower pace. However, when the movement sequence was relatively simple (1300-ms duration and three reversals) participants performed significantly better on the motor transfer test than on the spatial transfer test following only 99 trials of practice (Kovacs et al., 2009a). Experiments also indicated that the patterns of effector transfer were different for the same sequence when the sequence was produced using either pre-planned or on-line control strategies.

Thus, it appears that the amount of practice as proposed by Hikosaka et al. (1999; also see Bapi et al., 2000, 2006) and also the task characteristics and associated control processes play a role in determining the effectiveness of the coordinate system used in coding sequential movements at a particular stage of practice with the coordinate system determining the effector transfer conditions that lead to more effective transfer. Relatively simple movements (e.g., limited number of sequential elements, short durations) that are produced via pre-planned control processes appear to be best coded in motor coordinates with effector transfer and inter-limb practice enhanced when these coordinates are reinstated on the transfer test. Alternatively, more complex movement sequences (e.g., increased number of sequential elements and longer durations) that involve greater on-line control are best coded in visual-spatial coordinates with transfer and inter-limb practice enhanced when this coordinate system is reinstated on effector transfer tests. The coordinate

\section{REFERENCES}

Abrahamse, E., Jimenez, L., Verwey, W., and Clegg, B. (2010). Representing serial action and perception. Psychon. Bull. Rev. 17, 603-623.

Badets, A., and Blandin, Y. (2004). The role of knowledge of results frequency in learning through observation. J. Mot. Behav. 36, 62-70.

Bapi, R. S., Doya, K., and Harner, A. M. (2000). Evidence for effector independent and dependent representations and their differential time course of acquisition during motor sequence learning. Exp. Brain Res. 132, 149-162.

Bapi, R. S., Miyapuram, K. P., Graydon, F. X., and Doya, K. (2006). fMRI investigation of cortical and subcortical networks in the learning of abstract and effector-specific representations of motor sequences. Neuroimage 32, 714-727.

Bird, G., and Heyes, C. (2005). Effectordependent learning by observation of a finger movement sequence. J. Exp. Psychol. Hum. Percept. Perform. 31, 262-275.

Bischoff-Grethe, A., Goedert, K. M., Willingham, D. T., and Grafton, S. T. (2004). Neural substrates of responsebased sequence learning using fMRI. J. Cogn. Neurosci. 16, 127-138.
Blandin, Y., and Proteau, L. (2000). On the cognitive basis of observational learning: development of mechanisms for the detection and correction of errors. Q. J. Exp. Psychol. 53, 846-867.

Boutin, A., Fries, U., Panzer, S., Blandin, Y., and Shea, C. H. (2010). Role of action observation and action in sequences learning and coding. Acta Psychol. 135, 240-251.

Buccino, G., Binkofski, F., Fink, G. R., Fadinga, L., Gallese, R. J., Seitz, R. J., Zilles, K., Rizzolatti, G., and Freund, H.-J. (2001). Action observation activates premotor and parietal areas in a somatotopic manner: an fMRI study. Eur. J. Neurosci. 13, 400-404.

Criscimagna-Hemminger, S.E., Donchin, O., Gazzaniga, M. S., and Shadmehr, R. (2003). Learned dynamics of reaching movements generalize from dominant to non-dominant arm. J. Neurophysiol. 89, 168-176.

Decety, J., Grèzes, J., Costes, N., Perani, D., Jeannerod, M., Procyk, E., Grassi, F., and Fazio, F. (1997). Brain activity during observation of actions. Influence of action content and subject's strategy. Brain 120, 1763-1777.

Fadiga, L., Fogassi, L., Pavesi, G., and Rizzolatti, G. (1995). Motor facilitation during action observation:

system used to code moderately difficult movements may be determined by the availability of intrinsic and extrinsic feedback that is available for on-line control.

These findings may prove important in explaining the inconsistencies in the extant effector transfer literature where in many cases only one effector transfer test was administered (see Abrahamse et al., 2010 for review). If the effector transfer test was constructed such that the participant had to produce a mirror image of the practiced movement with the contralateral limb, we would predict more effective transfer if the representation used to produce the movement was coded in motor coordinates than if the representation was coded in visual-spatial coordinates. If the transfer test requires the same spatial movement pattern, for example, we would predict more effective transfer if the representation used to produce the movement was coded in visual-spatial coordinates than if the representation was coded in motor coordinates. What the present review highlights are some additional factors (number of reversals or elements, movement duration, control processes used) besides the amount of practice that may play a role in determining the coordinate system resulting in more effective transfer of movement sequences. It is also important to remember that not only effector transfer experiments, but within limb transfer experiments where either the display of stimuli, spatial pattern of the end effector, or limb position changes on the transfer test may benefit from considering the coordinate system used to code the movement sequence. Indeed, this latter type of experiments were initially used by Hikosaka et al. (1999), Bapi et al. (2000, 2006), and Willingham et al. (2000, Experiment 2), for example, to investigate the coordinate system used to code movement sequences.

a magnetic stimulation study. J. Neurophysiol. 73, 2608-2611.

Glover, S. (2004). Separate visual representations in the planning and control of action - response. Behav. Brain Sci. 27, 57-78.

Grafton, S. T., Hazeltine, E., and Ivry, R. B. (2002). Motor sequence learning with the nondominant left hand - a PET functional imaging study. Exp. Brain Res. 146, 369-378.

Grèzes, J., and Decety, J. (2001). Functional anatomy of execution, mental simulation, observation, and verb generation of actions: a meta-analysis. Hum. Brain Mapp. 12, 1-19.

Gruetzmacher, N., Panzer, S., Blandin, Y., and Shea, C. H. (2011). Observation and physical practice: coding of simple motor sequences. Q. J. Exp. Psychol. doi: 10.1080/17470218.2010.543286

Guiard, Y. (1993). On Fitts's and Hooke's laws: simple harmonic movement in upper-limb cyclical aiming. Acta Psychol. 82, 139-159.

Hikosaka, O., Nakahara, H., Rand, M. K., Sakai, K., Lu, X. F., Nakamura, K., Miyachi, S., and Doya, K. (1999). Parallel neural networks for learning sequential procedures. Trends Neurosci. 22, 464-471.
Hikosaka, O., Nakamura, K., Sakai, K., and Nakahara, H. (2002). Central mechanisms of motor skill learning. Curr. Opin. Neurobiol. 12, 217-222.

Hikosaka, O., Rand, M. K., Miyachi, S., and Miyashita, K. (1995). Learning of sequential movements in the monkey: process of learning and retention of memory. J. Neurophysiol. 74, 1652-1661.

Hikosaka, O., Sakai, K., Miyauchi, S., Takino, R., Sasaki, Y., and Putz, B. (1996). Activation of human presupplementary motor area in learning of sequential procedures: a functional MRI study. J. Neurophysiol. 76, 617-621.

Jordan, M. I. (1995). The organization of action sequences - evidence from a relearning task. J. Mot. Behav. 27, 179-192.

Keele, S. W., Jennings, P., Jones, S., Caulton, D., and Cohen, A. (1995). On the modularity of sequence representation. J. Mot. Behav. 27, 17-30.

Klapp, S. T. (1995). Motor response programming during simple and choice reaction time: the role of practice. $J$. Exp. Psychol. Hum. Percept. Perform. 21, 1015-1027.

Kovacs, A. J., Boyle, J., Grutmatcher, N., and Shea, C. H. (2010). Coding of 
on-line and pre-planned movement sequences. Acta Psychol. 133, 119-126.

Kovacs, A. J., Han, D.-W., and Shea, C. H. (2009a). The representation of movement sequences is related to task characteristics. Acta Psychol. 132, 54-56.

Kovacs, A. J., Muehlbauer, T., and Shea, C. H. (2009b). The coding and effector transfer of movement sequences. J. Exp. Psychol. Hum. Percept. Perform. 35, 390-407.

Krakauer, J.W., Ghilardi, M. F., and Ghez, C. (1999). Independent learning of internal models for kinematic and dynamic control of reaching. Nat. Neurosci. 2, 1026-1031.

Lange, K., Braun, C., and Godde, B. (2006). Coordinate processing during the left-to-right hand transfer investigated by EEG. Exp. Brain Res. 168, 547-556.

Lange, R. K., Godde, B., and Braun, C. (2004). EEG correlates of coordinate processing during intermanual transfer. Exp. Brain Res. 159, 161-171.

Mattar, A. A., and Gribble, P. L. (2005). Motor learning by observation. Neuron 46, 153-160.

Osman, M., Bird, G., and Heyes, C. (2005). Action observation supports effectordependent learning of finger movement sequences. Exp. Brain Res. 165, 19-27.

Panzer, S., Gruetzmacher, N., Krueger, M., Fries, U. and Shea, C. H. (2011). Aging effects in inter limb-practice and coding of movement sequences. Hum. Mov. Sci. doi: 10.1016/j. humov.2010.11.003
Panzer, S., Krueger, M., Muehlbauer, T., Kovacs, A. J., and Shea, C. H. (2009a). Inter-manual transfer and practice: coding of simple motor sequences. Acta Psychol. 131, 99-109.

Panzer, S., Muehlbauer, T., Krueger, M., Buesch, D., Naundorf, F., and Shea C. H. (2009b). Effects of interlimb practice on coding and learning of movement sequences. Q. J. Exp. Psychol. 62, 1265-1276.

Park, J.-H., and Shea, C. H. (2002). Effector independence. J. Mot. Behav. 34, 253-270

Park, J.-H., and Shea, C. H. (2003a). The independence of sequence structure and element production in timing sequences. Res. Q. Exerc. Sport 74, 401-420.

Park, J.-H., and Shea, C.H. (2003b). Effect of practice on effector independence. J. Mot. Behav. 35, 33-40.

Park, J.-H., and Shea, C. H. (2005). Sequence learning: response structure and effector transfer. Q. J. Exp. Psychol. 58, 387-419.

Rizzolatti, G., Fogassi, L., and Gallese, V. (2001). Neurophysiological mechanisms underlying the understanding and imitation of action. Nat. Neurosci. 2, 661-670.

Sakai, K., Hikosaka, O., Miyauchi, S., Takino, R., Sasaki, Y., and Putz, B. (1998). Transition of brain activation from frontal to parietal areas in visuomotor sequence learning. J. Neurosci. $18,1827-1840$.

Schmidt, R.A. (1975). A schema theory of discrete motor skill learning. Psychol. Rev. 82, 225-260.
Shea, C. H., Wulf, G., Park, J.-H., and Gaunt, B. (2001). Effects of an auditory model on the learning of relative and absolute timing. J. Mot. Behav. 33, 127-138.

Smiley-Oyen, A. L., and Worringham, C. J. (2001). Peripheral constraint versus on-line programming in rapid aimed sequential movements. Acta Psychol. 108, 219-245.

van Mier, H. I., and Petersen, S. E. (2006). Intermanual transfer effects in sequential tactuomotor learning: evidence for effector independent coding. Neuropsychology 44 939-949.

Verwey, W. B. (1994). Evidence for the development of concurrent processing in a sequential key pressing task. Acta Psychol. 85, 245-262.

Verwey, W. B. (1995). A forthcoming key press can be selected while earlier ones are executed. J. Mot. Behav. 27, 275-284.

Verwey, W. B., and Clegg, B. A. (2005). Effector dependent sequence learning in the serial RT task. Psychol. Res. 69, 242-251.

Whitacre, C., and Shea, C. H. (2002). The role of parameter variability on retention, parameter transfer, and effector transfer. Res. Q. Exerc. Sport 73, 47-57.

Wilde, H., Panzer, S., and Shea, C. H. (2008). The effects of sequence difficulty and practice on proportional and non-proportional transfer. Q. J. Exp. Psychol. 61, 1321-1339.

Wilde, H., and Shea, C. H. (2006). Proportional and non-proportional transfer of movement sequences. Q.J. Exp. Psychol. 59, 1626-1647.

Willingham, D. B., Wells, L. A., Farrell J. M., and Stemwedel, M. E. (2000). Implicit motor sequence learning is represented in response locations. Mem. Cognit. 28, 366-375.

Wright, D. L., Li, Y., and Coady, W. J. (1997). Cognitive processes related to the contextual interference and observational learning: a replication of Blandin, Proteau, and Alain (1994). Res. Q. Exerc. Sport 68, 106-109.

Conflict of Interest Statement: The authors declare that the research was conducted in the absence of any commercial or financial relationships that could be construed as a potential conflict of interest.

Received: 03 November 2010; accepted: 21 March 2011; published online: 08 April 2011.

Citation: Shea CH, Kovacs AJ and Panzer S (2011) The coding and intermanual transfer of movement sequences. Front. Psychology 2:52. doi: 10.3389/ fpsyg.2011.00052

This article was submitted to Frontiers in Movement Science and Sport Psychology, a specialty of Frontiers in Psychology. Copyright (c) 2011 Shea, Kovacs and Panzer. This is an open-access article subject to a non-exclusive license between the authors and Frontiers Media SA, which permits use, distribution and reproduction in other forums, provided the original authors and source are credited and other Frontiers conditions are complied with. 